\title{
Dual Homing based Link Level Failure Recovery in Mobile Access Network
}

\author{
K. Rajalakshmi and Krishna Gopal \\ Department of Computer Science and Engineering \\ Jaypee Institute of Information Technology \\ Noida, India \\ k.rajalakshmi@jiit.ac.in, krishna.gopal@jiit.ac.in
}

\begin{abstract}
The mobile access network designing provides an effective designing platform for entire mobile network. The mobile access network designing involves optimal assignment of communication link between components of the access network, satisfying various technical constraints of the network. Moreover, components of mobile access network are vulnerable to failure due to technical and environmental factors. Therefore, it is a critical problem, to define an effective mechanism for handling these faults at minimum cost while designing the mobile access network. In this paper we propose, heuristics based optimal fault tolerant algorithm to minimize the overall cost of mobile access network designing along with fault tolerant mechanism. Mathematical programming is used to formulate the problem. Further, six faults mechanisms are identified based on failure types, failure components at various access network level. These fault tolerant mechanism uses dual homing as the solution, in which case secondary link exists as backup along with primary communication between network components during link assignment of access network. The algorithm produces an optimal fault tolerant assignment of link satisfies the capacity and connectivity constraints of access network components. The algorithm outperforms well both in the smaller network and larger network.
\end{abstract}

Keywords: Optimization, fault tolerance, dual homing, mathematical programming, capacity constraint, connectivity constraint, primary link, secondary link

\section{Introduction}

Various factors like tremendous growth in mobile technology, mobile subscribers, consumers expectation, cheaper cost, best quality of service, on demand applications opens up necessity for effective designing of mobile network. The conventional wired access network parameters like protocols, methodology, quality, fairness, and performance metrics are no more sufficient for mobile network, due to its typical problems like limited bandwidth, power consumption and mobility. Adding to it, the structure of the mobile network itself is fat and strictly hierarchical network. Therefore, those approaches for wired network designing are not suitable for mobile network. There is a need for continuous improvement in methodology to be incorporated at mobile access network level.

Mobile access network is an important part within the entire mobile network designing. In mobile network designing, it is important to take care of the mobile access network designing, so that the overall mobile network designing is effective. The optimal designing of mobile access network, impacts the overall cost of the mobile network designing. Similarly the performance of mobile access network, impacts the overall performance of the mobile network. Some of the performance metrics of the mobile network are survivability, reliability, dependability, scalability, robust. This paper focuses on survivability of the mobile access network, in which, the fault tolerance to the link 
level failure is discussed. Mobile access network is prone to frequent failure due technical and environmental reasons. The nodes of the access network topology are NodeBs, RNCs, and MSCs. The link or edges are the physical transmission cables through which the nodes communication each other. Access network is broadly classified as NodeB level, RNC level and MSC level. Table1 gives the various failures occurring at different level of the access layer of the wireless cellular network. Two types of failures of wireless access network, namely node failure and link failure. In node failure, the components like NodeB, RNC and MSC may fail. In link failure, the transmission links between the components fails, even though the nodes are active. As given in [3], the survivability strategy for node or link failure is multi-homing of nodes. Oriol et. al., [1] incorporates coverage of mobile cells using sub optimal solution for UMTS network. Ekkalul et. al., [2] address $3 \mathrm{G}$ cellular network optimization. The reliability is the main focus of paper, mechanisms for failure prone optical access network is addressed. Tipper et. al., [3] discuss techniques for fault tolerance in wireless access network. Global Planning involving cell level, access network level, core network level is discussed by Liu et. al., [4]. Novillo et. al., [4] discusses load balancing strategies by optimal assignment of base station in mobile access network. Further performance analysis on transport level load balancing is incorporated in [10-13]. Mobile Access network consist of various component level like NodeB level, RNC level, MSC level. Varsheny et. al., [5], discuss infrastructure oriented multilevel fault tolerance in wireless networks. Performance evaluation of $3 \mathrm{G}$ cellular access network is been carried out by Ekkkal et. al., [6]. The cost optimization incorporating reliability of access network is analyzed. Hybrid genetic algorithm is used by Salcedo et. al., [7] to perform optimal switch allocation in mobile access network.

Mobile access network is prone to frequent faults like link fault and node fault due to software or hardware failures [15-17]. In this paper, the target is, to recover link from fault, therefore, whenever a fault occurs, then the necessary mechanism is taken to perform fault recovery. Here we use the mechanism called dual homing [18-19] in the link level failure recovery at mobile access network as given in table 1. In this paper, we are proposing six different mechanisms of fault recovery at link level.

Table 1. Failure Scenarios in Mobile access Network

\begin{tabular}{|l|l|}
\hline Components & NodeB, RNC, MSC \\
\hline Communication Link & Wire line links and/or terrestrial microwave \\
\hline Failure Scenario & $\begin{array}{l}\text { (1) Link Failure : Loss of NodeB to RNC link } \\
\text {; Loss of RNC to MSC link } \\
\text { (2) Node Failure: Loss of NodeB, RNC }\end{array}$ \\
\hline Potential Impact & $\begin{array}{l}\text { Partial/full service loss in cluster of cells, } \\
\text { increased traffic in cells adjacent to failure }\end{array}$ \\
\hline Survivable Strategy & $\begin{array}{l}\text { (1) Spare NodeB-RNC link } \\
\text { (2) Multi homing NodeB-RNC }\end{array}$ \\
\hline
\end{tabular}

The outline of the paper is section II discusses the mathematical programming of the given problem of optimal assignment using fault tolerant mechanism in mobile access network designing. Section III discusses the six types of fault tolerance mechanism; section IV explains the optimal fault tolerant algorithm in detail. Further Section V discusses the experimentation and analysis on various results obtained for different network instances and Section VI concludes the paper. 


\section{Mathematical Programming}

Given, $\alpha$ represents distance matrix between NodeB and RNC, $\beta$ represents binary flow matrix between NodeB and RNC, $\lambda$ represents the required capacity of NodeB, $\omega$ and represents residual capacity of RNC. The basic minimization cost function is given by equation (1). The objective is to find feasible solution for assignment of NodeBi to a $\mathrm{RNCj}$ such that overall link assignment cost is minimum. Further, the connectivity constraint given in equation (2) ensures that not more than one link is active between a particular NodeB $_{i}$ and a particular RNCj. The capacity constraint given in equation (3) ensures RNCs serving capacity is within the limits of requesting capacity of NodeBs.

Minimize $\quad \delta_{i}^{(j)}=\sum_{i=1}^{J} \sum_{j=1}^{J} \alpha_{i}^{(j)} \beta_{i}^{(j)}$

Subject to:

$\sum_{\mathrm{i}=1}^{\mathrm{I}} \beta_{\mathrm{is}}^{(\mathrm{j})} \cdot \mathrm{v}_{\mathrm{is}}=1$ for $\mathrm{j}=1,2, \ldots . \mathrm{J}$

$\sum_{\mathrm{i}=1}^{\mathrm{I}} \lambda_{\mathrm{is}}^{(\mathrm{j})} \beta_{\mathrm{is}}^{(\mathrm{j})} \leq \omega_{\mathrm{j}}$ for $\mathrm{j}=1,2, \ldots, \mathrm{J}$

$\beta_{\text {is }} \in\{0,1\}$

$\mathrm{v}_{\text {is }} \in\{0,1\}$

$s \in\{1,2\}$

The parameter $\mathrm{v}$ is used to indicate whether dual homing is incorporated by the algorithm while handling faults. The parameter $\mathrm{s}$ is used to indicate whether primary link $(s=1)$ is connected between NodeB and RNC, or secondary link $(s=2)$ is connected.

\section{Fault Tolerance Mechanism}

The effective solution for fault tolerant mobile access network designing is dual homing of NodeBs. By using a dual-homed mobile access network, in which each NodeB can reach at least two RNCs and can find the optimal route configurations, by minimizing the connection cost at RNC, without violating any of the capacity constraints. Fault tolerance is achieved by incorporating parameters like homing of access network nodes, type of failure encountered in the links and RNC assignment during failure. The types of link failures are single link failure and multiple link failure. In single link failure, any one of the available links connecting different NodeBs to a particular RNC is fails. In multiple link failure, more than one links connected to RNC to NodeBs are failed. By using dual homing, the problem of link failure can be resolved. The link failure between NodeB and RNC can be resolved using dual homing. In dual homing more than one link, namely primary link and secondary link, exists between NodeB and RNC. Upon using secondary link during fault tolerance, the secondary link can be assigned as dedicated one to one back up link or else as shared secondary link. While assigning NodeBs to RNC, the assignment could be Inter RNC and Intra RNC levels. Thus based on these parameters like homing type, failure type, RNC assignment type, six different classes of fault tolerance mechanism has been identified as given in Figure 1.

\section{(i) Intra RNC dedicated back up single link failure}

Here target is single link failure, whenever single link failure occurs between RNC and NodeB, then the proposed fault tolerance mechanism performs by assigning dedicated back up link as survivability support. The assignment of back link is between NodeBs within the single roof of RNC. Hence its intra RNC dedicated single link failure. 


\section{(ii) Intra RNC dedicated back up multiple link failure}

Whenever multiple link failures occur between RNC and NodeB, then the proposed fault tolerance mechanism performs by assigning dedicated back up links as survivability support. The assignment of back links is between NodeBs within the single roof of RNC. (iii) Intra RNC shared back single link failure.

\section{(iv) Intra RNC shared back multiple link failure}

Dedicatedly assigning back up links as discussed in above cases (i) and (ii) can be improved further by using shared links. In order to achieve better performance and optimization in link assignment, the shared back up is best suitable. The shared link reduces the cost factor further at the RNC node.

\section{(vi) Inter RNC multiple link failure}

Above case (i) to (iii) are possible failure scenario and their fault tolerance mechanism. There exists an exceptional failure scenario, the intra RNC assignment fails due to capacity constraint equation (3). In such cases, the proposed fault tolerant mechanism uses Inter RNC assignment, where the failed NodeB will be assigned to other suitable RNC for survivability.

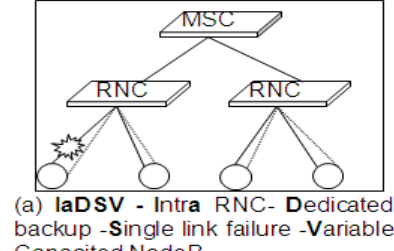
Capacited NodeB

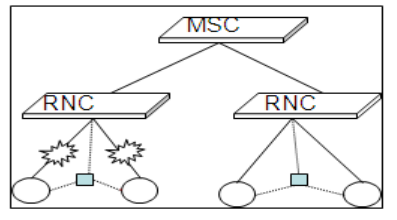

(d) laSMV - Intra RNC- Shared backup-Multiple link failure Variable Capacited NodeB

Where $\quad$

$\square \rightarrow$ Ethernet like Switch; rfc4968 for shared link model

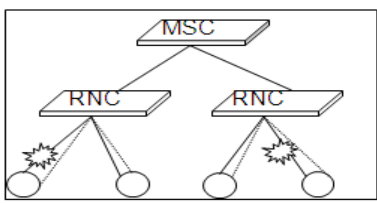

(b) IaDMV - Intra RNC- Dedicated backup -Multiple link failure Variable Capacited NodeB

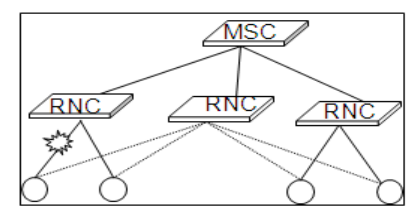

(e) les SV - Inter RNC- Single link failure-Variable Capacited NodeB R.

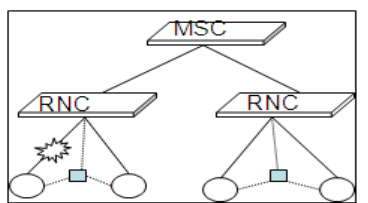

(c) lassV - Intra RNC- Shared backup - Single link failure Variable Capacited NodeB

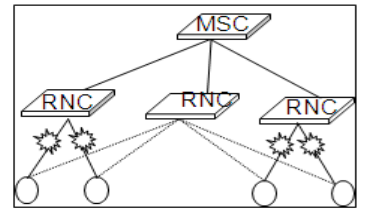

(f) le SMV - Inter RNC-Multiple link failure-Variable Capacited NodeB

\section{Figure 1. Fault Tolerance Mechanism in Dual Homed Mobile Access Network}

\section{Optimized Fault Tolerant Algorithm (OFTA)}

\section{Heuristic Algorithm}

Iterative local search is a simple and powerful metaheuristics among all other heuristic algorithms as the algorithm uses efficient local search and avoids gravel into local optimal by using effective perturbation operator. Basically two operators are used in ILS algorithm, namely, local search operator and perturbation operator. Whenever local search gets trapped into a local optimal solution, a perturbation operator is applied. A fresh starting point for further local search is obtained. However, the generated starting point must suitably be in proximity of solution within search space. Pseudo code for ILS is given in Figure 2. Algorithm begins with applying a local search on general initial solution $\mathrm{P}$ and produces new local optimal solution $\mathrm{P}^{*}$. 
Local Search improves a solution in the search space. When the local search is stuck, the locally optimal solution $\mathrm{P}^{*}$ is mutated by a move in a neighborhood different from the one already used by the local search. Perturbation kicks current local solution $\mathrm{P}^{*}$ and generate an intermediate solution P'. The resulting solution of perturbation P' is the new starting solution for the local search that takes it again to another new local optimum $\mathrm{P}^{*}$ '. Using proximate optimality principle, if $\mathrm{P}^{\prime}$ is close to $\mathrm{P}^{*}$ in Perturbation, then the new local optimal solution generated in local search will also be in proximity of the current local optimal solution.

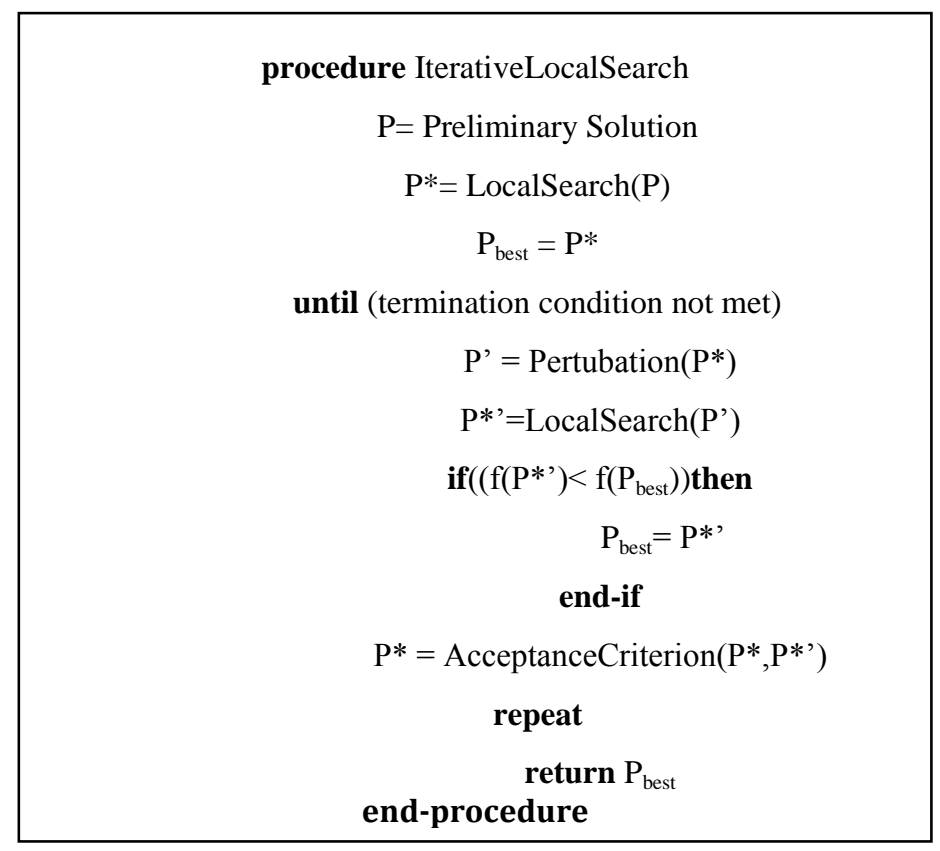

Figure 2. Pseudo-code for Iterative Local Search

Finally an acceptance criterion decides which of the two locally optimal solutions, $\mathrm{P}^{*}$, and $\mathrm{P}^{*}$, obtained from previous step is to be select as a starting point for the next perturbation step. If $\mathrm{P}^{*}$ ' win $\mathrm{P}^{*}$ in acceptance criterion, then $\mathrm{P}^{*}$ ' will replace $\mathrm{P}^{*}$ and become current optimal solution, otherwise the search remains at the previous optimal solution $\mathrm{P}^{*}$. The terminating condition for this algorithm is number of iterations. The major impulse in ILS Algorithm lays in carrying out a randomized walk within the search space of the local optima with respect to some local search algorithm.

\section{Optimized Fault Tolerant Algorithm (OFTA)}

Now that basic metaheuristic algorithm is defined, we incorporated optimized fault tolerant algorithm within this. An Optimized fault tolerant algorithm for link assignment of mobile access network consist of three stages like primary link assignment, failure ink assignment, estimation of cost and computation time as explain below.

Definition\#1 (Valid link Assignment): Given a binary variable $x$, for every $p$ belongs to the set of primary links $P$ and for every $s$ belong to the set of secondary links $S$, iff $p \neq s$, and $\sum_{n=1}^{N}\left(x_{p}^{n}+x_{s}^{n}\right)=N$, then exists a valid link assignment between any two nodes, each at different level in the access network. Example: Let $\mathrm{N}=8$, there exists 8 RNCs. Each RNC has a primary link and a secondary link. There are 8 primary links and 8 secondary links. To know that there exist a faulty link, 


$$
\begin{aligned}
& \sum_{n=1}^{N}\left(x_{p}^{n}+x_{s}^{n}\right) \\
& =\left(x_{p}^{1}+x_{s}^{1}\right)+\left(x_{p}^{2}+x_{s}^{2}\right)+\left(x_{p}^{3}+x_{s}^{3}\right)+\ldots+\left(x_{p}^{8}+x_{s}^{8}\right) \\
& =(1+0)+(1+0)+(1+0)+(1+0)+(1+0)+(1+0)+(1+0)+(1+0) \\
& =8
\end{aligned}
$$

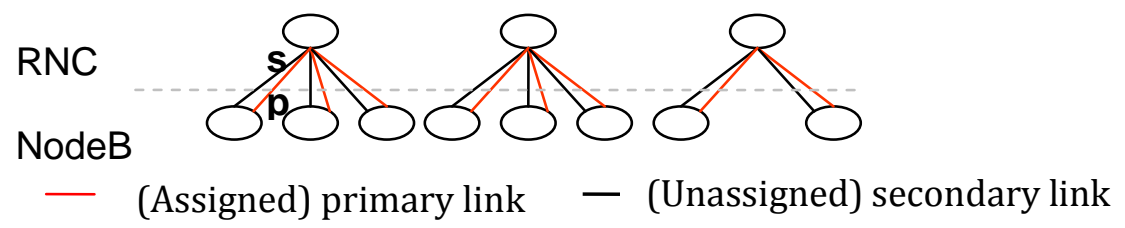

Definition\#2 (Link Failure): Given a binary variable $x, \mathrm{n}$ represent node in set $\mathrm{N}$ in cellular access network, for every $p$ belongs to the set of primary links $P$ and for every $s$ belong to the set of secondary links $S$, then if $\sum_{n=1}^{N}\left(x_{p}^{n}+x_{s}^{n}\right)<N$, then there exist link failure between any two nodes in the access network.

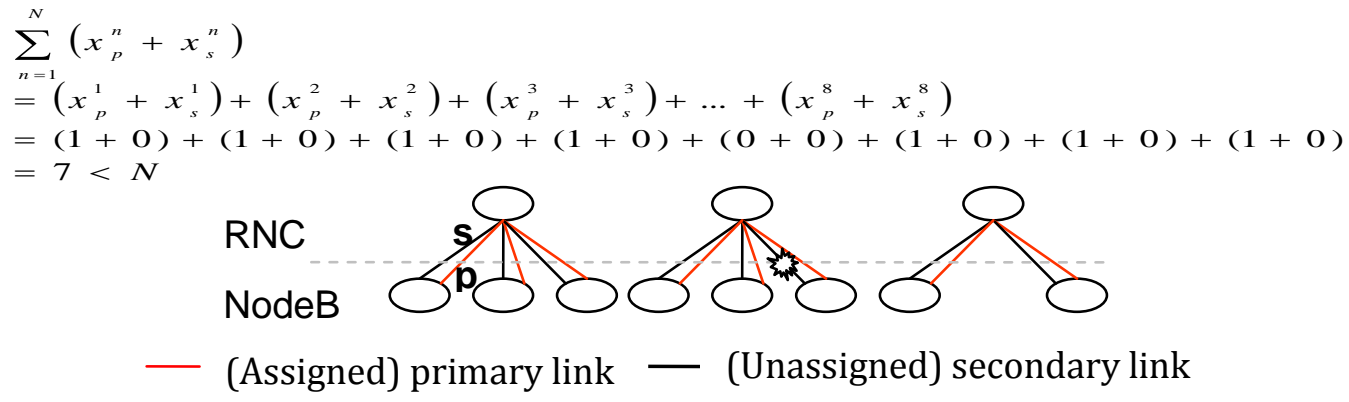

Definition\#3 (Invalid link Assignment): Given a binary variable $x$, for every $p$ belongs to the set of primary links $P$ and for every $s$ belong to the set of secondary links $S$, iff $p=s$, and $\sum_{n=1}^{N}\left(x_{p}^{n}+x_{s}^{n}\right)>N$, then there do not exist a valid link assignment between any two nodes, each at different level, in the access network.

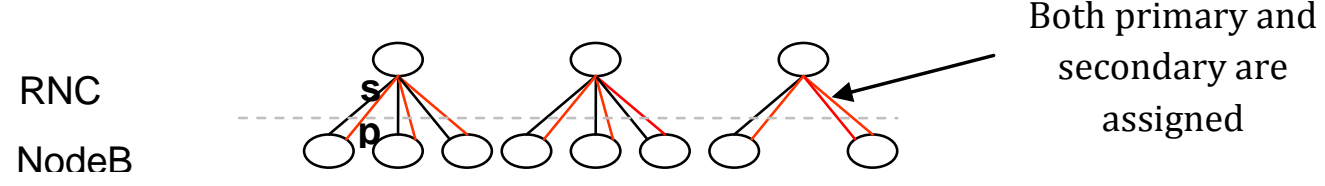

- (Assigned) primary link _ - (Unassigned) secondary link

$$
\begin{aligned}
& \sum_{n=1}^{N}\left(x_{p}^{n}+x_{s}^{n}\right) \\
& =\left(x_{p}^{1}+x_{s}^{1}\right)+\left(x_{p}^{2}+x_{s}^{2}\right)+\left(x_{p}^{3}+x_{s}^{3}\right)+\ldots+\left(x_{p}^{8}+x_{s}^{8}\right) \\
& =(1+0)+(1+0)+(1+0)+(1+0)+(1+0)+(1+0)+(1+0)+(1+1) \\
& =9>N
\end{aligned}
$$

Link assignment: To begin with find the minimum distance between NodeBs and RNCs using Euclidean distance formula. Find the first minimum distanced RNC and also second minimum distance RNC between the NodeBs and RNCs. Check by assign the first minimum distanced RNC to NodeB. From equation (5), the variable $\mathrm{v}$ represents single homing or dual homing; s represents primary or secondary links assignment. 
Intra RNC Primary link assignment (given $v=0, s=1$ ): Check primary link of first minimum distanced RNC to NodeB is not failed. Check the connectivity constraint by assigning only one RNC to NodeB. Check the residual capacity of the RNC (capacity constraint using equation 3). If residual capacity of the RNC is within the limit, then first minimum distance RNC is assigned to NodeB. The record of RNCs is updated.

1. Procedure_link assignment

1.1. Finding the minimum distance between NodeBs and RNCs using Euclidean distance

1.2. Find the first minimum distanced RNC and second minimum distance RNC between the NodeBs and RNCs

1.3. Check by assign the first minimum distanced RNC to NodeB

1.3.1. Check (primary link of first minimum distanced RNC to NodeB != failure)

1.3.1.1. Check the connectivity constraint by assigning only one RNC to NodeB

1.3.1.2. Check the residual capacity of the RNC (capacity constraint)

1.3.1.3. If residual capacity of the RNC is within the limit

1.3.1.4. Then assign the first minimum distance RNC to NodeB , \& update the record of RNCs

1.3.2. Check (secondary link of first minimum distanced RNC to NodeB != failure)

1.3.2.1. Check the connectivity constraint by assigning only one RNC to NodeB

1.3.2.2. Check the residual capacity of the RNC (capacity constraint)

1.3.2.3. Then assign the first minimum distance RNC to NodeB \& update the record of RNCs

1.4. Check by assigning the second minimum distanced RNC to NodeB

1.4.1. Check the connectivity constraint by assigning only one RNC to NodeB

1.4.2. Check the residual capacity of the RNC (capacity constraint)

1.4.3. If residual capacity of the RNC is within the limit

1.4.4. Then assign the first minimum distance RNC to NodeB \& update the record of RNCs

2. Procedure_validating assignment

2.1. Check (valid assignment using definition \#1) go to step 3.

2.2. Else (Check invalid assignment using definition\#2),

2.2.1. Then goto step (1) link assignment procedure

2.2.2. Perform valid assignment procedure goto step (2)

2.3. Else (Check link failure using definition \#3), then repeat link assignment procedure step (1).

2.3.1. Then goto step (1) link assignment procedure

2.3.2. Perform valid assignment procedure go to step (2)

3. Procedure_Estimation of Cost \& Computation Time

3.1. Using the objective function formulated mathematically.

3.2. Output the minimized cost of fault tolerant access network

3.3. Output consumed computation time

Figure 3. Pseudo Code for Link Assignment Procedure in OFTA

Intra RNC Secondary link assignment (given $v=1, s=2$ ): In case of primary link failure, secondary link is assigned either as dedicated or as shared link. In Single link failure as given in figure $1(\mathrm{a}-\mathrm{b})$, NodeB with link failure is assigned with dedicated secondary link to RNC. In Multiple link failure as given in Figure 1 (c-d), NodeBs with link failure are 
assigned with shared secondary link to RNC. Check whether, secondary link of first minimum distanced RNC to NodeB is functional. If so, check whether connectivity constraint by assigning only one RNC to NodeB. Check the residual capacity of the RNC (capacity constraint).

\section{Inter RNC link Assignment.}

In case of first minimum distanced RNC, has not adequate residual capacity for requested NodeB capacity then inter RNC link assignment is incorporated. Check by assigning the second minimum distanced RNC to NodeB. Check the connectivity constraint by assigning only one RNC to NodeB. Check the residual capacity of the RNC (capacity constraint). If residual capacity of the RNC is within the limit of required NodeB capacity, then assign the second minimum distance RNC to NodeB \& update the record of RNCs.

\section{Validating assignment:}

Check valid assignment using definition \#1, if so estimates Cost \& Computation. Else check invalid assignment using definition\#2. Then repeat link assignment procedure. Perform valid assignment procedure. Else Check link failure using definition \#3, then repeat link assignment procedure. Then go to step of link assignment procedure. If done, then perform valid assignment procedure.

Estimation of Cost \& Computation Time: Output the minimized cost of fault tolerant access network using equation (1) - (6). Output consumed computation time.

\section{Example:}

Example link assignment in a $24 \times 4$ mobile access network with 24 NodeBs and 4 RNCs is illustrated in Figure 2. The NodeBs XY coordinates are given in column $2 \& 3$. The Primary link capacity and secondary link capacity are given in column $4 \& 5$. By using the above algorithm, (i) Intra RNC Dedicated back single link failure is exhibited by nodeB\#2 is assigned to secondary link (ii) Intra RNC Dedicated back Multiple link failure is exhibited by NodeBs $(2,14,22)$ being assigned with dedicated secondary link(iii) Intra RNC shared back single link failure is exhibited by NodeB\#1 assigned to intra RNC \#3

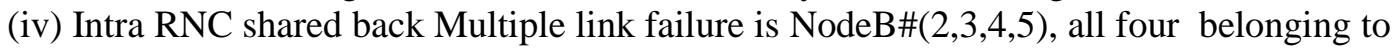
RNC \#1 (v) Inter RNC single link failure is exhibited by nodeB\#1 is assigned to inter RNC\#3 (vi) Inter RNC multiple link failure as given in figure nodeBs\#1 to intra RNC\#3 , nodeB\#22 is assigned to intra RNC\#4. 


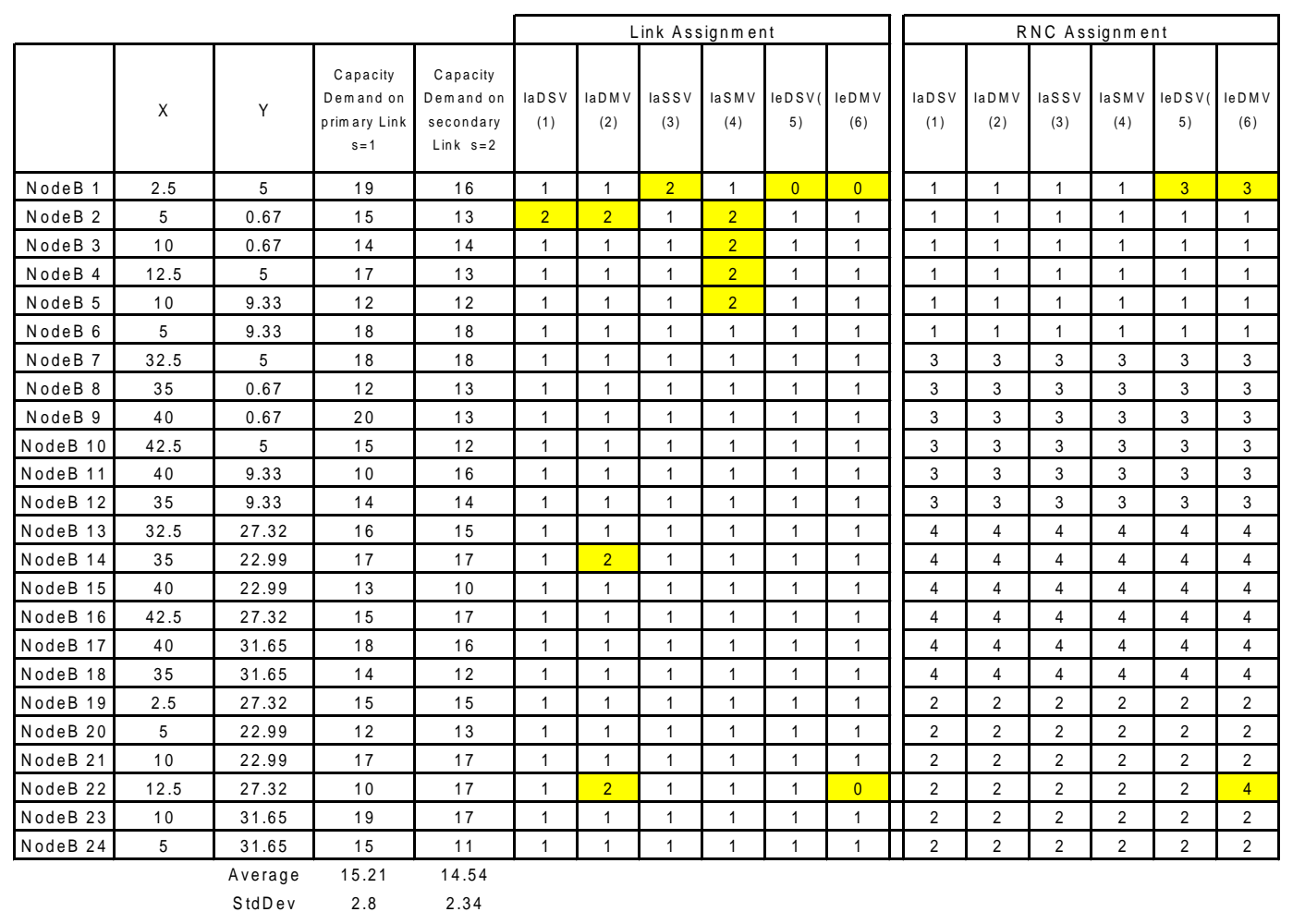

\begin{tabular}{|c|c|c|c|}
\hline RNC 1 & 7.5 & 5 & 159 \\
\hline RNC 2 & 7.5 & 27.32 & 189 \\
\hline RNC 3 & 22.5 & 13.66 & 169 \\
\hline RNC 4 & 37.5 & 27.32 & 147 \\
\hline
\end{tabular}

\section{Figure 4. Fault Tolerance Mechanism in 24x4 Mobile Access Network}

\section{Experimentation}

Experiment has been conducted on a Dell N5110 Model with Intel(R) Core(TM) i72630QM CPU@2.00GHZ; RAM 6.00GB, 64bit Operating System. Optimized Fault Tolerant Algorithm (OFTA) had been implemented using visual studio C++ programming. A network instance is represented by $\mathrm{NxM}$, where $\mathrm{N}$ represents number of NodeBs and $\mathrm{M}$ represents number of RNS in mobile access network. For example, $24 \mathrm{x} 4$ networks is meant by 24 NodeBs with 4 RNCs in a mobile access network. The input to the algorithm is NxM integer matrix $\left(\alpha_{i}^{j}\right)$ as given in equation (1), representing distance between NodeB to the given RNC. The NxM binary flow matrix $\left(\beta_{i}^{j}\right)$, indicates whether there exists a link between given NodeB and RNC. Non Zero Value of the binary matrix indicates link existence; zero value indicates no link existence between particular $N{ }^{2} B_{i}$ and $\mathrm{RNC}_{\mathrm{j}}$. The vector $\left(\lambda_{i}\right)$ indicates the capacity of NodeBs primary link when the variable $s=1$, otherwise it represents the capacity of secondary link at $s=2$. The vector $\left(\omega_{j}\right)$ represents the residual capacity of RNCs that is available for allocation to requesting NodeBs. Table 2 provides various network instances and RNC capacity. The output is an optimal assignment of $\mathrm{NodeB}_{i}$ to $\mathrm{RNC}_{\mathrm{j}}$ at minimal distance cost, with fault tolerance to the link failure; ensuring technical constraints like capacity and connectivity are satisfied. The experiment has been carried out for smaller network medium size network and larger network.

The experiment consists of two parts namely, cost optimization part, fault tolerance part. The optimal assignment of NodeBs to various RNCs along with connectivity and capacity constraint is carried based on equation (1-6) and then fault tolerance is carried using Optimized Fault Tolerant Algorithm (OFTA). 
Table 2. Network Instance \& its RNC Capacity

\begin{tabular}{|c|c|c|c|}
\hline Network Id & NodeB & RNC & $\begin{array}{c}\text { Total RNC } \\
\text { Capacity (Mbps) }\end{array}$ \\
\hline 1 & 25 & 5 & 65 \\
\hline 2 & 50 & 10 & 136 \\
\hline 3 & 75 & 15 & 185 \\
\hline 4 & 100 & 20 & 263 \\
\hline 5 & 125 & 25 & 320 \\
\hline 6 & 150 & 30 & 390 \\
\hline
\end{tabular}

Consider the specific case of $24 \times 4$ mobile access network, means 24 NodeBs and 4 RNCs. As given in Table 1, the ideal total capacity of 4 RNC is $664 \mathrm{Mbps}$, with average of $166 \mathrm{Mbps}$, the average capacity consumption for various fault tolerance mechanism are $91 \mathrm{Mbps}$ for IaDSV, 92.5Mbps in case of IaDMV,91.5Mbps for IaSSV, 96.75Mbps for IaSMV, $92.5 \mathrm{Mbps}$ for IaDSV and $91.75 \mathrm{Mbps}$ for IeDMV , graphically depicted as Figure 3.

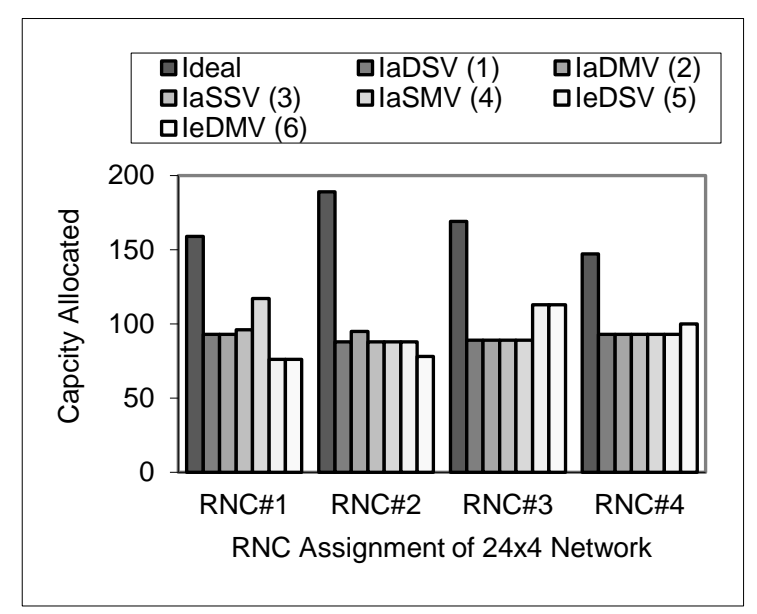

Figure 2. Average Capacity Allocated in 24x4 Mobile Access Network for Six Cases of Fault Tolerance Mechanism

From Table 3, the total average of utilized RNC capacity for all six cases of fault tolerance mechanism is $56 \%$ and the average percentage of residual capacity for all six fault tolerance mechanism is $\sim 44 \%$.

Table 3. Analysis of Mobile Access Network (24x4) for Six Cases of Fault Tolerance Mechanism

\begin{tabular}{|c|c|c|c|c|c|c|c|}
\hline Parameters & Ideal & $\begin{array}{c}\text { IaDSV } \\
(1)\end{array}$ & $\begin{array}{c}\text { IaDMV } \\
(2)\end{array}$ & $\begin{array}{c}\text { IaSSV } \\
(3)\end{array}$ & $\begin{array}{c}\text { IaSMV } \\
(4)\end{array}$ & $\begin{array}{c}\text { IeDSV } \\
(5)\end{array}$ & $\begin{array}{c}\text { IeDMV } \\
(6)\end{array}$ \\
\hline RNC\#1 & 159 & 93 & 93 & 96 & 117 & 76 & 76 \\
\hline RNC\#3 & 169 & 89 & 89 & 89 & 89 & 113 & 113 \\
\hline RNC\#4 & 147 & 93 & 93 & 93 & 93 & 93 & 100 \\
\hline $\begin{array}{c}\text { Average } \\
\text { allocated }\end{array}$ & 166 & 90.75 & 92.5 & 91.5 & 96.75 & 92.5 & 91.75 \\
\hline
\end{tabular}




\begin{tabular}{|c|c|c|c|c|c|c|c|}
\hline $\begin{array}{c}\text { capacity } \\
\text { (Mbps) }\end{array}$ & & & & & & & \\
\hline $\begin{array}{c}\text { Total RNCs } \\
\text { capacity } \\
\text { (Mbps) }\end{array}$ & 664 & 363 & 370 & 366 & 387 & 370 & 367 \\
\hline $\begin{array}{c}\text { Utilized RNC } \\
\text { capacity (\%) }\end{array}$ & 55 & 56 & 55 & 58 & 56 & 55 \\
\hline $\begin{array}{c}\text { Residual } \\
\text { capacity of } \\
\text { RNC (\%) }\end{array}$ & 45 & 44 & 45 & 42 & 44 & 45 \\
\hline
\end{tabular}

The Ideal average capacity utilized by RNCs of the mobile access network using random assignment greedy algorithm is $75 \%$ without any link failure. The minimum capacity utilized is $73 \%$ till maximum capacity utilization of $77 \%$. The overall residual capacity of the RNCs is $27 \%$, with minimal residual capacity of $25 \%$ for $50 x 10$ networks till maximum residual capacity of $29 \%$ for $25 \times 5$ networks.

As given in Table 4, the proposed Optimized Fault Tolerant Algorithm (OFTA), outperform the random assignment algorithm in both single link failure and multiple link failure. For single link failure, the total average capacity of RNCs utilized by optimal fault tolerant assignment is $\sim 75 \%$, whereas the total average of residual capacity in RNCs is achieved as $\sim 27 \%$. This shows the outperform s of OFTA algorithm compared to random assignment algorithm.

Table 4. Comparative Analysis of Random Assignment and OFT Algorithm

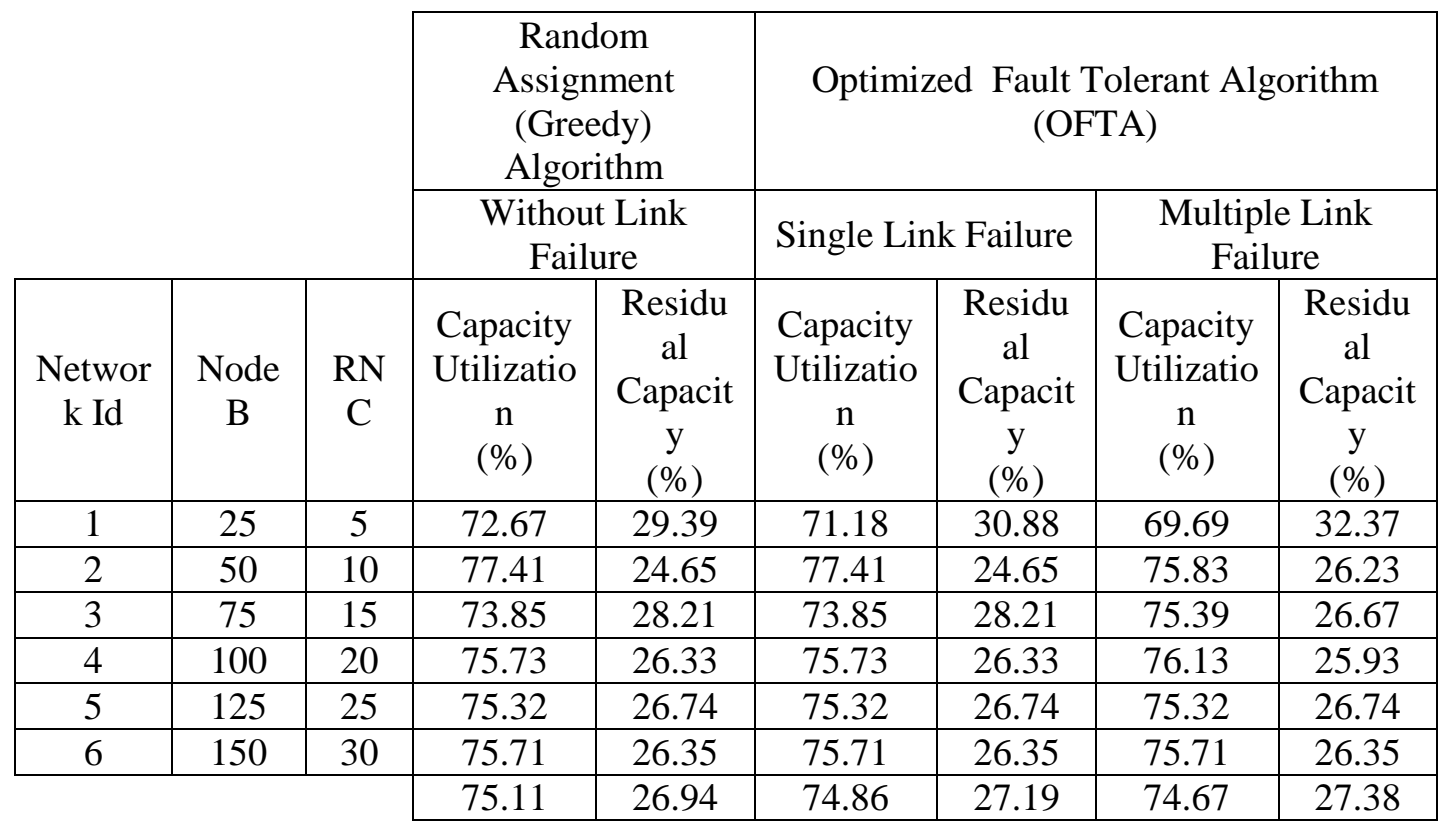

In case of Multiple link failure, the algorithm produces total average RNC capacity utilized as $\sim 75 \%$ and total average RNC residual capacity as $\sim 27 \%$. It is to note that, the algorithm does not suffer due to multiple link failure handling. The important outcome of proposed algorithm is that assignment of optimal RNCs to various Nodes is performed without much parameter deviation between single and multiple link failures. Also the varying size of network from smaller $(25 \times 5)$ till larger network $(150 \times 30)$ are handled effectively by the algorithm. 
Table 5. Comparison of Computation Time

\begin{tabular}{|c|c|c|c|c|c|}
\cline { 4 - 6 } \multicolumn{2}{l|}{} & $\begin{array}{c}\text { Random } \\
\text { Assignment } \\
\text { (Greedy) } \\
\text { Algorithm }\end{array}$ & $\begin{array}{c}\text { Optimal Fault Tolerant Algorithm } \\
\text { (OFTA) }\end{array}$ \\
\hline $\begin{array}{c}\text { Netwrk } \\
\text { Id }\end{array}$ & NodeB & RNC & $\begin{array}{c}\text { Without link } \\
\text { failure } \\
\text { Processing Time } \\
(\mathrm{sec})\end{array}$ & $\begin{array}{c}\text { Single link } \\
\text { failure } \\
\text { Processing Time } \\
(\mathrm{sec})\end{array}$ & $\begin{array}{c}\text { Multiple link } \\
\text { failure } \\
\text { Processing Time } \\
(\mathrm{sec})\end{array}$ \\
\hline 1 & 25 & 5 & 1.614 & 0.114 & 0.114 \\
\hline 2 & 50 & 10 & 2.792 & 0.208 & 0.192 \\
\hline 3 & 75 & 15 & 5.902 & 0.286 & 0.302 \\
\hline 4 & 100 & 20 & 6.058 & 0.411 & 0.411 \\
\hline 5 & 125 & 25 & 9.583 & 0.567 & 0.599 \\
\hline 6 & 150 & 30 & 9.817 & 0.786 & 0.771 \\
\hline
\end{tabular}

The comparison of computation time for various mobile access network, exhibits the Optimal fault tolerant algorithm performs random greedy assignment algorithm as given in Table 5. The without link failure using greedy algorithm consumes $6 \mathrm{sec}$ for various network models considered, whereas, the average computation time consumed by single link failure is $0.4 \mathrm{sec}$. And for the multiple link failure the algorithm produces average computation time as $0.4 \mathrm{xec}$. The significance is that the algorithm performs well for both single link failure as well as multiple link failure effectively. Further, the computation time remains approximately same for smaller network and larger network.

\section{Conclusion}

Mobile access network designing involves optimal assignment of link between NodeBs and RNCs. In this paper effective algorithm, namely optimal fault tolerant algorithm is proposed. The effect of this algorithm in solving link failures in access network is studied. Six different fault tolerance mechanisms are incorporated and their outcomes are analyzed. The proposed algorithm also outperforms than random assignment algorithm. The total average capacity allocation to RNC is achieved to be $\sim 75 \%$ with residual capacity of RNC $\sim 27 \%$ both single link failure and multiple link failures. The computation time also exhibits significant improvement compared with random algorithm. The random algorithm without link failure consumes $\sim 6 \mathrm{sec}$ for optimal assignment, where as the proposed OFT algorithm consumes $\sim 0.4 \mathrm{sec}$ for bother cases of single link failure and multiple link failure. Also the algorithm performs effectively in terms of computation time and capacity allocation for both smaller and larger network size of mobile access network designing.

\section{References}

[1] O. Sallen, "A Roadmap from UMTS Optimization to LTE Self-Optimization", IEEE Communications Magazine, (2011), pp. 172-182.

[2] D. Tipper, T. Dalhberg and H. Shin, "Providing Fault Tolerance in Wireless Access Networks," IEEE Communications Magazine, vol. 40, no. 1, (2002), pp. 58-64.

[3] S. Liu and M. Hilaire, "A Genetic Algorithm for the Global Planning Problem of UMTS Networks", GLOBECOM 2010, (2010), pp. 1-5.

[4] F. Novillo, H. Galeana, A. Lainz, R. Ferrus and J. Olmos, "Performance analysis of transport and radio load balancing strategies for BS assignment in mobile access networks", IEEE 19th International Symposium on Personal, Indoor and Mobile Radio Communications, (2008), pp. 1-6.

[5] U. Varshney and A. D. Malloy, "Multilevel Fault Tolerance in Infrastructure-Oriented Wireless Networks: Framework and Performance Evaluation", International Journal of Network Management, vol. 16, no. 5, (2006), pp. 351-374. 
[6] E. Eksook and C. Prommak, "Reliability Optimization for 3G Cellular Access Network", International Journal of Engineering and Applied Sciences, vol. 5, no. 7, pp. 445-449, (2009).

[7] S. Salcedo-Sanz, J. A. Portilla-Figueras, E. G. Ortiz-García, A. M. Pérez-Bellido, C. Thraves, A. Fernández-Anta and X. Yao, "Optimal switch location in mobile communication networks using hybrid genetic algorithms", Applied Soft Computing, (2008), pp. 1486-1497.

[8] D. R. Din, Y. Chiu, "A genetic algorithm for solving virtual topology reconfiguration problem in survivable WDM networks with reconfiguration constraint", Computer Communications, vol. 31, no. 10, (2008), pp. 2520-2533.

[9] B. Krishnamachari, S. Wicker, "Base station location optimization in cellular wireless networks using heuristic search algorithms", Soft Computing in Communications, Springer, (2003), pp. 201-220.

[10] M. St-Hilaire and S. Liu, "Comparison of different meta-heuristics to solve the global planning problem of UMTS networks", Computer Networks, vol. 55, no. 12, (2011), pp. 2705-2716.

[11] M. St-Hilaire, "A New Approximative Algorithm for the Expansion Problem of 3G Networks", Information Systems and Operational Research (INFOR), vol. 47, no. 2, (2010), pp. 105-115.

[12] S. Liu and M. Hilaire, "A Genetic Algorithm for the Global Planning Problem of UMTS Networks", GLOBECOM 2010, (2010), pp. 1-5.

[13] A. Ju"ttner, Orba'n and Z. Fiala, "Two new algorithms for UMTS access network topology design", European Journal of Operational Research, vol. 164, (2005), pp. 456-474.

[14] S. Chamberland, "An efficient heuristic for the expansion problem of cellular wireless networks", Computers and Operations Research, vol. 31, no. 11, (2004), pp. 1769-1791.

[15] G. Mateus and R. V. Fanqueira, "Model and heuristic for a generalizated access network design problem”, Telecommunication Systems, vol. 15, (2000), pp. 257-271.

[16] P. Kubat, J. M. Smith and C. Yum, "Design of Cellular Networks with Diversity and Capacity Constraints", IEEE Transaction on Reliability, vol. 49, no. 2, (2000), pp. 165-175.

[17] F. Houeto, S. Pierre, R. Beaubrun and Y. Lemieux, "Reliability and Cost Evaluation of ThirdGeneration Wireless Access Network Topologies: A Case Study", IEEE Trans. On Reliability, vol. 51, no. 2, (2002), pp. 229-239.

[18] A. Snow, U. Varshney and A. Malloy, "Reliability and Survivability of Wireless and Mobile Networks," IEEE Computer, vol. 33, no. 7, (2000), pp. 49-55.

[19] A. Dutta and P. Kubat, "Design of partially survivable networks for cellular telecommunication systems", European Journal of Operational Research, vol. 118, (1999), pp. 52-64. 
International Journal of Advanced Science and Technology Vol.79 (2015) 